\title{
Nível de Ruído de Peças de Mão de Alta Velocidade Usadas em Odontologia como Fator de Risco Auditivo
}

\author{
Noise Level of High-Speed Handpieces Used in Dentistry \\ as Hearing Hazard Factor
}

\author{
Augusto Henrique Alves de Oliveira ${ }^{1}$ \\ Cristiskis Mikaelle Gonçalves de Lima² \\ Clarisse Maria Queiroz Couto ${ }^{2}$ \\ Camila Gonçalves $\mathrm{Cruz}^{2}$ \\ Francisco Jadson Lima ${ }^{1}$
}

\section{RESUMO}

Introdução: O ruído é um sinal acústico descrito como som indesejável ou desagradável. A exposição prolongada a níveis elevados de ruído configura um risco físico ocupacional relevante. Objetivos: Esta pesquisa teve como objetivos: avaliar o nível de ruído produzido por canetas de alta rotação utilizadas em odontologia; comparar os resultados com o limite máximo recomendado e níveis toleráveis conforme legislação; comparar resultados entre marcas e modelos. Materiais e Métodos: 0 nível de ruído de 152 turbinas foi medido com um decibelímetro digital, posicionado em uma câmara acústica. Resultados: O nível de pressão sonora produzido pelas peças de mão de alta velocidade avaliadas teve média de $62,92 \pm 2,99 \mathrm{~dB}$; Vinte e três amostras $(15,54 \%)$ apresentaram ruído acima de $65 \mathrm{~dB}$, enquanto $125(84,45 \%)$ não ultrapassaram o nível máximo de conforto acústico recomendado pela NR-17, apesar de terem atingidos resultados bem próximos do limite. Canetas de marcas e modelos diferentes não apresentaram diferenças significantes de níveis de ruído $(p=0,25)$. Conclusão: $O$ nível de pressão sonora provocado pelo uso de canetas de alta rotação situa-se próximo do limite máximo do conforto acústico quando avaliado isoladamente. Canetas de marcas e modelos diferentes não apresentaram diferenças significantes de níveis de ruído.

DESCRITORES: Ergonomia. Odontologia. Perda Auditiva. Ruído Ocupacional. Medição de Ruído.

\begin{abstract}
Introduction: Noise is an acoustic signal described as undesirable or unpleasant sound. Prolonged exposure to high noise levels is a relevant occupational physical hazard. Objectives: The aims of this research was: to evaluate the noise level produced by high speed hand pieces used in Dentistry; to compare the results with the recommended maximum limit and tolerable levels according to legislation; to compare results between brands and models. Material and Methods: The noise level of 152 turbines was measured with a digital sound level meter, positioned in an acoustic chamber. Results: The sound pressure level produced by the samples reached a mean of $62.92 \mathrm{~dB}(\mathrm{~A})$ and standard deviation of 2.99; Twenty-three samples (15.54\%) presented noise above $65 \mathrm{~dB}$, while $125(84.45 \%)$ did not exceed the maximum level of acoustic comfort recommended by the NR-17, although results were very close to the limit. High speed hand pieces of different brands and models did not present significant differences in noise levels $(p=0.25)$. Conclusion: The sound pressure level caused by the use of high-speed Handpieces is close to the maximum limit of acoustic comfort when evaluated in isolation. Handpieces of different brands and models showed no significant differences in noise levels.
\end{abstract}

DESCRIPTORS: Ergonomics. Dentistry. Hearing Loss. Occupational Noise. Noise Measurement.

\footnotetext{
Professor do curso de Odontologia do Centro Universitário Doutor Leão Sampaio - UNILEÃO. Juazeiro do Norte - Ceará - Brasil.
}

2. Acadêmica do curso de Odontologia do Centro Universitário Doutor Leão Sampaio - UNILEÃO. Juazeiro do Norte - Ceará - Brasil. 
A dontologia foi se modernizando ao longo dos anos, concomitantemente com o aumento da exposição do dentista a vários tipos de riscos ocupacionais no local de trabalho, que podem ser físicos, químicos, biológicos, ergonômicos, entre outros ${ }^{1,2}$. Um destes grandes avanços na odontologia foi a criação de um dispositivo rotatório conhecido como caneta de alta rotação que é utilizado para remoção de tecido cariado e restaurações insatisfatórias. Contudo, o ruído causado pelo instrumento ainda incomoda sobremaneira os profissionais. Embora a idade e a susceptibilidade individuais possam desempenhar um papel significativo, a intensidade, a distância da fonte e a duração total do ruído demonstram repercussão na audição ${ }^{3,4}$.

Do ponto de vista da acústica física, som é uma perturbação vibratória em um meio elástico, que produza sensação auditiva, e ruído é um sinal acústico aperiódico, originado da superposição de vários movimentos de vibração com diferentes frequências que não apresentam relação entre si. Ou seja, a definição de ruído é englobada pela definição de som ${ }^{5,6}$. Psicologicamente, enquanto o som é utilizado para descrever sensações prazerosas, o ruído é usado para descrever sons indesejáveis ou desagradáveis, o que traz um aspecto de subjetividade à sua definição ${ }^{7}$.

A exposição prolongada a níveis elevados de ruído de frequência irregular pode ser percebida como desconfortável ou mesmo dolorosa. O ruído produzido pelas canetas de alta-rotação, também conhecidas como "turbina", não é apenas desagradável aos pacientes, dentistas e equipe, configurando um risco físico ocupacional relevante, que pode estar relacionado com acidentes e comprometimentos orgânicos diversos tais como hipertensão arterial, depressão, estresse, aumento da tensão muscular, incapacidade de concentração e perda auditiva induzida por ruído (PAIR) $)^{6,8}$.

Considerando-se a importância de praticar a odontologia de forma segura para o paciente e o profissional, os objetivos desta pesquisa foram: avaliar o nível de ruído produzido por canetas de alta rotação utilizadas em odontologia; comparar os resultados com o limite máximo recomendado e níveis toleráveis conforme legislação; e comparar resultados entre marcas e modelos.

\section{MATERIAIS E MÉTODOS}

Trata-se de um estudo experimental de observação direta, com fins descritivos e analíticos. Esta pesquisa recebeu dispensa do Comitê de
Ética em Pesquisa pois analisa somente equipamentos. Registrou-se o ruído produzido por 152 canetas de alta rotação, de diferentes marcas e modelos, de acadêmicos de odontologia e alguns critérios foram aplicados para seleção das amostras.

Os critérios de inclusão foram: peças de mão de alta velocidade com apresentação de fábrica original em perfeito estado de funcionamento; aquelas que os proprietários concedessem os instrumentos e assinassem um termo de cessão voluntária, sem obter vantagem alguma por isso. Foram excluídos deste estudo: peças de mão de alta velocidade que não estivessem funcionando no momento da experiência; que tivessem sido modificadas ou consertadas, mesmo que em assistência técnica especializada.

O nível de ruído das peças de mão de alta velocidade foi aferido por meio de um decibelímetro digital. É um medidor de pressão sonora portátil, modelo DEC-490, fabricante INSTRUTHERM São Paulo - SP Brasil, número de série $13022700967536 / 13014693$ com precisão de \pm $1,4 \mathrm{~dB}$, com microfone condensador eletreto de $1 / 2$ polegada com uma resolução de $0.1 \mathrm{~dB}$. Suas dimensões são $76 \mathrm{~mm}$ X 50mm X $27,8 \mathrm{~cm}$, peso de $350 \mathrm{~g}$, e capacidade de memória de até 32.000 registros, compatível com o sistema operacional Windows XP ou posterior. Pode-se classificar como um tipo contínuo de ruído por apresentar pouca variação nos valores aferidos. Trabalhamos as leituras em nível de escala automática $(30 \mathrm{~dB}$ a $130 \mathrm{~dB}$ ). O medidor foi posicionado na curva de ponderação "A" e a constante de tempo em lento (SLOW = pressão de som RMS em 1 segundo) de acordo com a metodologia da NR 15.

Para este experimento, uma caixa de teste foi construída em madeira macia (pinho), nas dimensões de $50 \mathrm{~cm} \times 50 \mathrm{~cm} \times 50 \mathrm{~cm}$, e recebeu duplo revestimento acústico, a primeira camada de $5 \mathrm{~mm}$ de poliestireno expandido, o ISOFAMA São Paulo - SP Brasil, coberto por uma segunda camada de poliuretano macio de $20 \mathrm{~mm}$, PROESPUMA São Paulo - SP Brasil. Esses materiais foram escolhidos por suas propriedades como excelente isolamento térmico-acústico de uso generalizado na indústria e na construção. O objetivo do uso da caixa acústica foi de avaliar o ruído emitido por peças de mão de alta velocidade sem a influência de outras fontes. Durante o experimento, o número de pesquisadores na sala foi reduzido ao mínimo. Esta metodologia foi inspirada/adaptada na câmara acústica do estudo de Mueller e colaboradores $3,9,10$.

Duas pequenas aberturas foram feitas na 
caixa, em lados opostos de $15 \mathrm{~cm}$ do canto superior esquerdo e a outra a $15 \mathrm{~cm}$ do canto inferior direito. No primeiro orifício foi posicionado o microfone do decibelímetro que foi usado no estudo. Uma vez instalado, o dispositivo permaneceu fixo na mesma posição até o final da coleta de dados. $\mathrm{Na}$ segunda abertura foram inseridas as canetas de alta rotação (espécimes). Esta disposição coloca o emitente (turbinas) e o receptor (microfone) a uma distância próxima da distância entre a peça de mão e a orelha do dentista em uma situação clínica usual.

As peças de mão de alta rotação foram acionadas por aproximadamente 40 segundos. A irrigação foi desligada e nenhuma ponta ou broca foi acoplada ${ }^{11}$. Para a nossa avaliação, consideramos os 20 segundos em que os instrumentos estivessem funcionando a toda velocidade. Os dados foram registrados individualmente em planilhas por meio de software específico. Por se tratarem de dados paramétricos (numéricos), adotou-se o teste ANOVA para comparação entre os grupos, procurando por diferenças entre as amostras e o pós-teste $Q$ de Tukey de comparação par-a-par.

\section{RESULTADOS}

Quatro amostras do fabricante GNATUS, Ribeirão Preto - SP Brasil, sendo duas do modelo SL 30 FG (Friction-Grip) e duas do modelo SL 30 PB (Press-Button) ambos TB (Acople Borden), não foram considerados na análise estatística devido ao número

As demais amostras foram divididas em grupos de acordo com o modelo e o fabricante. Noventa e quatro peças de mão da marca KAVO, Joinville - SC Brasil, foram identificados os modeIos EXTRA TORQUE 505 FG TB $(n=14)$, EXTRA TORQUE 505c PB TB $(n=67)$ EXTRA TORQUE 605 FG TB $(n=7)$ e EXTRA TORQUE 605C PB
TB $(n=6) ; 12$ peças da marca DABI ATLANTE, Ribeirão Preto SP - Brasil, modelo SILENT PB TB modelo; E 42 peças DENTFLEX, modelo SIGMA AIR 3S PB TB modelo. Os modelos foram avaliados em grupos específicos como mostrado na Tabela 2 e Figura1.

Realizou-se a análise da relação entre a variável dependente (ruído) e as independentes (fabricantes e modelos), por meio da construção de modelos de análise de variância (ANOVA) e pós-teste $Q$ de Tukey. Os resultados são apresentados na Tabela 3 e Tabela 4, onde não foram observadas diferenças estatísticas entre os grupos pesquisados $(p>\alpha)$.

\section{DISCUSSÃO}

Sobre a metodologia adotada, pode-se apontar como limitações ou potencial fonte de viés a falta de padronização das amostras. Algumas das turbinas avaliadas eram seminovas, nunca usadas em situações clínicas, outras apresentavam até três anos de uso. Contudo, canetas de alta rotação com diferentes tempos de uso sendo avaliadas permitem uma interpretação das variações que podem surgir com o tempo pelo uso/mau uso, lubrificação, esterilização, quedas, entre outros.

A legislação brasileira aborda o ruído em ambientes odontológicos de forma indireta, e porque não dizer, subestima o risco físico ao qual profissionais da odontologia estão expostos diariamente. Por meio da Portaria nº. 3.214/1978 foram aprovadas as Normas Regulamentadoras (NRs) - do Capítulo V, Título II, da Consolidação das Leis do Trabalho $(\mathrm{CLT})$ relativas à Segurança e Medicina do Trabalho.

O exercício de trabalho em condições de insalubridade é regulado pela NR - 15 que determina que os níveis de ruído contínuo ou intermitente devem ser medidos em decibéis $(\mathrm{dB})$ com instru-

Tabela 1 - Valores máximos, mínimos e média de ruídos em decibéis das amostras excluídas da análise estatística.

\begin{tabular}{c|c|c|c|c|c}
\hline Fabricante & Modelo & Data/Horário & Max & Min & Média \\
\hline GNATUS & SL 30 PB TB & $14-09-2016,20: 24: 21$ & 65,1 & 62,1 & 63,87 \\
GNATUS & SL 30 PB TB & $20-09-2016,20: 16: 25$ & 63,4 & 60,5 & 62,45 \\
GNATUS & SL 30 FG TB & $21-09-2016,17: 17: 44$ & 61,9 & 60,8 & 61,19 \\
GNATUS & SL 30 FG TB & $21-09-2016,17: 23: 55$ & 57,7 & 56,1 & 56,83 \\
\hline
\end{tabular}


Tabela 2 - Contagem e percentual das amostras em relação com o nível de conforto acústico recomendado; valores de média, desvio padrão, mínimo e máximo de todos e cada modelo analisado.

\begin{tabular}{l|c|c|c|c|c|r|r|r}
\hline & $\boldsymbol{n}$ & $\boldsymbol{\%}$ & $\boldsymbol{n}$ & \multicolumn{1}{c}{$\boldsymbol{\%}$} & Média & \multicolumn{1}{c}{ DP } & \multicolumn{1}{c}{ Min } & \multicolumn{1}{c}{ Max } \\
\hline Extra Torque 505 & 1 & $4,30 \%$ & 13 & $10,40 \%$ & 62,85 & 1,64 & 58,6 & 65,2 \\
Extra Torque 505C & 7 & $30,40 \%$ & 60 & $48,00 \%$ & 62,78 & 2,13 & 59,6 & 70,12 \\
Extra Torque 605 & 4 & $17,40 \%$ & 3 & $2,40 \%$ & 64,22 & 3,1 & 57,91 & 66,74 \\
Extra Torque 605C & 0 & $0,00 \%$ & 6 & $4,80 \%$ & 59,58 & 3,67 & 53,79 & 64,13 \\
Sigma Air & 10 & $43,50 \%$ & 32 & $25,60 \%$ & 63,69 & 3,79 & 54,8 & 74,72 \\
Silent & 1 & $4,30 \%$ & 11 & $8,80 \%$ & 62,64 & 3,77 & 55,41 & 71,1 \\
\hline Total & 23 & $15,54 \%$ & 125 & $84,45 \%$ & 62,92 & 2,9934 & 53,79 & 74,72 \\
\hline
\end{tabular}

Tabela 3 - Teste One-way ANOVA de comparação intra e inter grupos $(p=0,25)$.

\begin{tabular}{l|r|r|r|r}
\hline \multicolumn{1}{c|}{ Grupo } & Contagem & \multicolumn{1}{c}{ Soma } & Média & Variância \\
\hline Kavo 505 e 505c & 81 & 5086,03 & 62,79 & 4,19 \\
Kavo 605 E 605c & 13 & 807,02 & 62,08 & 16,21 \\
Dabi atlante Silent & 12 & 751,72 & 62,64 & 14,22 \\
Dentflex Sigma Air & 42 & 2675,01 & 63,69 & 14,38 \\
\hline
\end{tabular}

\begin{tabular}{l|r|r|r|r|r|c}
\hline Fonte da variação & \multicolumn{1}{c|}{$\boldsymbol{S} Q$} & \multicolumn{1}{c|}{$\boldsymbol{g} \boldsymbol{l}$} & \multicolumn{1}{c|}{$\boldsymbol{M Q}$} & $\boldsymbol{F}$ & valor-P & $\boldsymbol{F}$ crítico \\
\hline Entre grupos & 36,04 & 3 & 12,01 & 1,36 & 0,26 & 2,67 \\
Dentro dos grupos & 1275,47 & 144 & 8,86 & & & \\
Total & 1311,51 & 147 & & & $\alpha=0,05$ & \\
\hline
\end{tabular}

Tabela 4 - Pós teste $Q$ de Tukey de comparação par-a-par.

$\underline{Q}$ de Tukey

\begin{tabular}{l|c|c|c|c}
\hline & KAVO 505 e 505c & KAVO 605 E 605c & Dabi Atlante Silent & Dentflex Sigma Air \\
\hline Kavo 505 e 505c & & 0,8709 & 0,9986 & 0,7692 \\
Kavo 605 E 605c & 1,08 & & 0,9303 & 0,3087 \\
Dabi atlante Silent & 0,2231 & 0,8565 & & 0,6753 \\
Dentflex Sigma Air & 1,365 & 2,445 & 1,588 & \\
\hline
\end{tabular}


FIGURA 1 - Gráfico Boxplot modelos em decibéis.

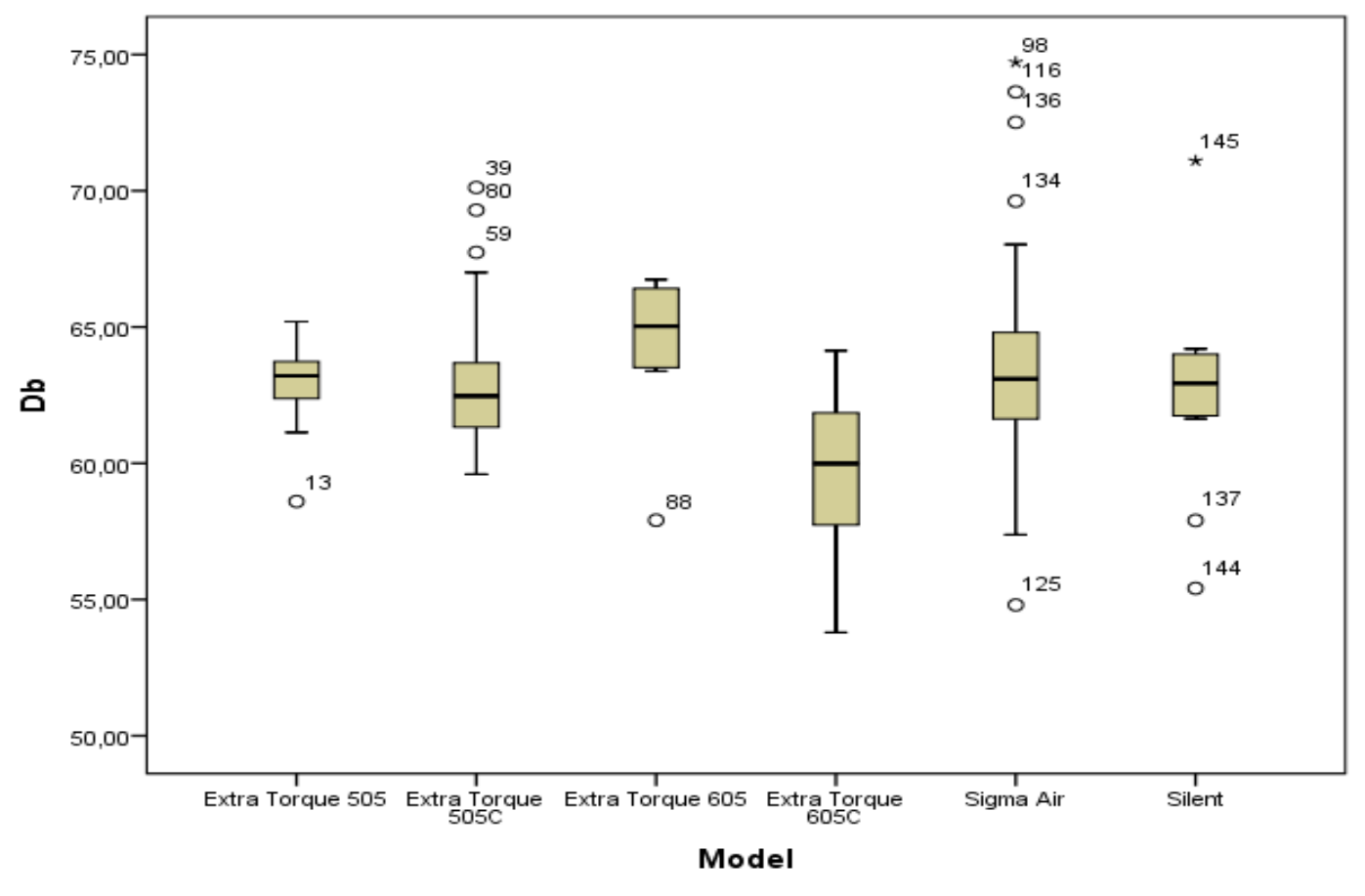

mento de nível de pressão sonora operando no circuito de compensação "A" e circuito de resposta lenta (slow). As leituras devem ser feitas próximas ao ouvido do trabalhador. Os Limites de tolerância só são consideradas a partir de $85 \mathrm{~dB}(\mathrm{~A})$ com no máximo 8 horas de exposição diária permissível, e considerando-se 5 decibéis como incremento de duplicação de dose. Ou seja, a cada cinco decibéis, o tempo máximo de exposição deve ser reduzido pela metade10,12. A norma de higiene ocupacional NHO 01 - "Avaliação da Exposição Ocupacional ao Ruído" (2001) trouxe como uma das principais modificações a adoção do valor "3" como incremento de duplicação de dose, que na NR -15 era de "5" 2 .

Equipamentos de proteção auditiva são recomendados na NR - 6 apenas contra níveis de pressão sonora superiores ao estabelecido na $N R$ - 15, ou seja, acima de $85 \mathrm{~dB}(\mathrm{~A})$. Os níveis médios de ruído em ambientes com instrumentos rotatórios como consultórios e laboratórios de prótese variam de 60 a $80 \mathrm{~dB}$, mas as pressões sonoras produzidas podem ser muito maiores. $A$ exposição ocupacional prolongada a ruídos representa um risco real de danos nos órgãos auditivos, particularmente dos trabalhadores que não usam equipamento de proteção individual $(\mathrm{EPI})^{13,14}$.

A finalidade da NR - 32 é estabelecer diretrizes básicas de implementação das medidas de proteção à segurança e à saúde dos trabalhadores dos serviços de saúde, bem como daqueles que exercem atividades de promoção e assistência à saúde em geral. No capítulo das disposições gerais, determina que os serviços de saúde devem atender a condições de conforto relativas aos níveis de ruído previstas na norma técnica NB 95 da ABNT15.

O projeto NB 95/1986 deu origem a norma NBR 10152/1987 que normatiza os níveis de ruído compatíveis com o conforto acústico em ambientes diversos, mas sem discutir questões relativas a riscos de dano à saúde em decorrência do ruído, e sem abordar diretamente o consultório odontológico. Para serviços de saúde em geral, a norma estabelece $45 \mathrm{~dB}(\mathrm{~A})$ como nível sonoro para conforto, enquanto $55 \mathrm{~dB}(\mathrm{~A})$ seria o nível sonoro aceitável para a finalidade. A norma reitera que níveis superiores aos estabelecidos são considerados de desconforto, contudo sem necessariamente implicar risco de dano à saúde ${ }^{16,17}$. 
Em contrapartida, pesquisas clínicas apontam um maior risco de perda auditiva em dentistas do que entre outros profissionais de saúde, e que este risco não é generalizado, mas era específico dos dentistas que frequentemente usavam equipamentos ruidosos (turbinas, por exemplo) durante a prática diária. Os dentistas com 10 ou mais anos de prática, utilizando rotineiramente equipamentos potencialmente ruidosos, podem estar em risco de perda auditiva. Uma forma de tentar minimizar este risco é proceder a manutenção diária e a substituição periódica destes instrumentais. Se somados, os ruídos produzidos por canetas de alta rotação somados a outros ruídos no consultório, facilmente alcançaria o limite da insalubridade 18.

A ergonomia (NR - 17) estabelece parâmetros para adaptação das condições de trabalho às características psicofisiológicas dos trabalhadores, de modo a proporcionar um máximo de conforto, segurança e desempenho eficiente. Ao ser atualizada pela Portaria MTPS $n^{\circ} .3 .751$, de 23 de novembro de 1990, incorporou os níveis de ruído de acordo com o estabelecido na NBR 10152, além de determinar que em locais de trabalho onde são executadas atividades que exigem solicitação intelectual e atenção constantes, mas que não apresentam equivalência ou correlação com aquelas relacionadas pela norma, como é o caso dos consultórios odontológicos, o nível de ruído aceitável para efeito de conforto será de no máximo $65 \mathrm{~dB}(\mathrm{~A})$ e a curva de avaliação de ruído (NC) de valor não superior a 60 dB19.

Os resultados desta pesquisa demonstram que o risco auditivo ocupacional inferido pelo uso de canetas de alta rotação deve ser comparado com o limite do conforto acústico recomendado pela legislação ${ }^{16,19}$. O nível de pressão sonora produzido pelas peças de mão de alta velocidade avaliadas teve média de $62,92 \mathrm{~dB}(\mathrm{~A})$, mediana $62,87 \mathrm{~dB}(\mathrm{~A})$ e desvio padrão de 2,99; Vinte e três amostras (15,54\%) apresentaram ruído acima de $65 \mathrm{~dB}$, enquanto $125(84,45 \%)$ não ultrapassaram o nível máximo de conforto acústico recomendado, apesar de terem atingidos resultados bem próximos do limite. Somados a outros ruídos no consultório, o conjunto facilmente ultrapassaria o limite da insalubridade10. Canetas de marcas e modelos diferentes não apresentaram diferenças significantes de níveis de ruído $(p=0,25)$.

Desde a década de 60 onde as turbinas de alta rotação forma introduzidas no mercado da odontologia, a maioria dos dentistas aceitou o ruído agudo, a fim de participar das vantagens distintas que as peças de mão acionadas por ar comprimido ofereciam. Os benefícios no controle do operador, a taxa de remoção da estrutura do dente e o trauma reduzido para o paciente superam em muito o aborrecimento resultante da exposição ao ruído agudo. Autores concordam em afirmar que o ruído produzido por instrumentos rotatórios de alta rotação não sejam capazes de provocar perda auditiva em cirurgiões dentistas, quando analisados isoladamente, já que os níveis deveram estar abaixo de $80 \mathrm{~dB}(\mathrm{~A})^{9,11}$.

É importante pontuar que os dados da maioria das pesquisas correspondem a equipamentos novos e em excelente estado de funcionamento. Sabe-se que o desgaste e a manutenção imprópria de peças de mão aumentam os níveis de ruído. Somam-se a isso outros ruídos que atuam concomitantemente com o da turbina afetando ainda mais o profissional. Barulhos produzidos pelo ar condicionado, amalgamadores, compressores, entre outros, muitas vezes passam despercebidos e não são considerados ruídos. O ruído ambiental, como o trânsito, vozes, telefone, companhia e o lazer se assomam a esta equação ${ }^{20}$.

Valores de pressão sonora superiores ao recomendado podem causar efeitos fisiológicos e psicológicos ${ }^{17}$. A literatura aponta que ruídos estão relacionados a doenças psicológicas, como estresse, irritabilidade e alterações no processo de comunicação e humor, sendo que o trabalhador pode desenvolver danos ao sistema neurológico, circulatório, digestório, endócrino, imunológico, vestibular, muscular, nas funções sexuais e reprodutivas e no sono. A literatura destaca ainda estresse, labirintite hipertensão arterial, perda do sono, impotência sexual, surdez bilateral ou unilateral entre outras ${ }^{21}$. Mais estudos são necessários para reforçar cientificamente estas associações ${ }^{22}$.

Corroborando com as informações evidenciadas no presente trabalho, outros autores relatam que o ruído no local de trabalho não somente causa perda auditiva, mas pode induzir injúrias e acidentes fatais que vão desde a perda de um membro do corpo até a vida ${ }^{23}$. Ainda nessa perspectiva, alguns outros trabalhos chamam a atenção para ação do ruído como fator causal de acidentes de trabalho uma vez que o mesmo pode promover distrações, aborrecimentos, déficit de atenção e estresse profissional ao ponto de contribuir com o aumento dos acidentes de trabalho ${ }^{24,25}$. Todas essas informações demonstram o quanto importante são trabalhos que buscam identificar o nível de ruídos produzidos por instrumentos de trabalho, neste trabalho especificamente o odontológico, uma vez que tais informações podem ser utilizadas 
na formulação de atitudes e ações preventivas.

A Perda Auditiva Induzida por Ruído (PAIR) é um dos agravos de notificação compulsória, apesar disso, estudos mostram que não é adequadamente notificada ${ }^{26}$. Os dados epidemiológicos sobre perda auditiva no Brasil são escassos e referem-se a determinados ramos de atividades e, portanto, não há registros epidemiológicos que caracterizem a real situação. Os dados disponíveis sobre as ocorrências dão uma ideia parcial da situação de risco relacionada à perda auditiva ${ }^{7}$.

A literatura relata evidências de perda auditiva em cirurgiões dentistas com uma média de 10 anos de profissão $0^{6,27}$ que podem ou não ser reversíveis, geralmente não estão associadas à dor, e podem permanecer não detectadas por anos. Um indivíduo pode experimentar uma perda auditiva de $28 \%$ (30 decibéis) antes de perceber o comprometimento. As principais queixas auditivas apresentadas são perda da capacidade auditiva, zumbido, intolerância a sons intensos e dificuldade de discriminação da fala ${ }^{4,28}$.

Alunos de Odontologia possuem entendimento sobre o ruído no dia-a-dia clínico e também sobre suas consequências ocupacionais sobre a saúde, contudo não demonstravam saber de forma eficaz sobre as medidas preventivas a serem tomadas para minimizar os efeitos do ruído excessivo29. A realização de um exame audiométrico inicial e outros periódicos para identificar as

\section{REFERÊNCIAS}

1. Brasil, Ministério da Saúde, Agência Nacional de Vigilância Sanitária. Serviços Odontológicos: Prevenção e Controle de Riscos. Vol. 1, Série A: Normas e Manuais Técnicos. Brasília: Editora MS; 2006. $152 \mathrm{p}$.

2. Brasil, Ministério do Trabalho e Emprego. Norma de Higiene Ocupacional 01-Avaliação da Exposição Ocupacional ao Ruído. São Paulo; 2001 p. 12-40.

3. Bahannan S, El-Hamid AA, Bahnassy A. Noise level of dental handpieces and laboratory engines. J Prosthet Dent. 1993;70(4):356-60.

4. von Krammer RK. High speed equipment and dentists' health. J Prosthet Dent. 1968;19(1):46-50.

5. Lacerda A, Melo SCS, Mezzadri SD, Zonta WG. Nível de pressão sonora de um consultório odontológico : uma análise ergonômica. Tuiuti Ciência e Cult. 2002;26(3):17-24.

6. Willershausen B, Callaway A, Wolf TG, Ehlers V, Scholz L, Wolf $\mathrm{D}$, et al. Hearing assessment in dental practitioners and other academic professionals from an urban setting. Head Face Med. 2014;10(1):1-7. injúrias auditivas provocadas pela exposição ao ruído excessivo no dia-a-dia clínico do profissional são recomendadas desde dos anos 80 . Os profissionais devem proceder à manutenção periódica seguindo as orientações dos fabricantes, afim de reduzir o risco à saúde provocado pelo ruído das peças de mãos de alta rotação utilizadas na odontologia ${ }^{30}$.

Além de avaliação e monitoramento da exposição ao ruído, são medidas de controle ambiental e organizativas como redução do ruído na emissão pela colocação de barreiras; materiais fonoabsorventes; redução da vibração nas estruturas, lubrificando corretamente as canetas de alta rotação; planejar alterações no esquema de trabalho ou das operações para permitir a redução de exposição ao ruído ${ }^{27}$.

\section{CONCLUSÃO}

Conclui-se que o nível de pressão sonora provocado pelo uso de canetas de alta rotação situa-se próximo do limite máximo do conforto acústico quando avaliado isoladamente. Canetas de marcas e modelos diferentes não apresentaram diferenças significantes de níveis de ruído $(p=0,034)$. Os dados sugerem que os fabricantes devem investir esforços no desenvolvimento de instrumentos menos ruidosos.

7. Brasil, Ministério da Saúde, Secretaria de Atenção à Saúde, Departamento de ações programáticas estratégicas. Perda Auditiva Induzida por Ruído ( Pair ). Brasília: Editora MS; 2006. p. 1-40

8. Silveira MFCR, Ricco FF, Lourenço MAJ, Ricco RAPO. Análise do ruído produzido pela caneta de alta rotação em diferentes procedimentos operatórios. Colloq Vitae. 2009;1(1):53-7.

9. Mueller HJ, Sabri ZI, Suchak AJ, McGill S, Stanford JW Noise level evaluation of dental handpieces. J Oral Rehabil. 1986;13(1970):279-92.

10. Brasil, Ministério do Trabalho e Emprego. NR 15 - Atividades e operações insalubres. 1978 p. 1-82.

11. Cantwell KR, Tunturi AR, Manny VR. Noise from high-speed dental handpieces. J Am Dent Assoc. 1960;61(5):571-7.

12. Soto JMOG, Saad IFSD, Giampaoli E, Fantazzini ML. Norma Regulamentadora (NR)-15, da Portaria n. 3.214, de 8.6.1978 do Ministério do Trabalho (atual Ministério do Trabalho e Emprego): Um pouco da sua história e considerações do grupo que a elaborou. Rev ABHO. 2010; $p$. $1-12$. 
13. Brasil, Ministério do Trabalho e Emprego. NR 6 - Equipamento de proteção individual. Portaria GM n.o 3.214, de 08 de junho de 1978 Brasil; 1978 p. 1-8.

14. Garus-Pakowska A, Leśniewska A, Gaszyńska E, Szatko F. Occupational exposure and health problems among Polish denturists: a population-based study in Lodz province. Int Dent J. 2016;66(4):237-46.

15. Brasil, Ministério do Trabalho e Emprego. NR 32 - Segurança e saúde no trabalho em serviços de saúde. 2005 p. $1-40$.

16. ABNT - Associação Brasileira de Normas técnicas. NBR 10152 - Níveis de ruído para conforto acústico. 1987 p. $1-4$.

17. Andrade KP, Oliveira LLA, Souza RP, Matos IM. Medida do nível de ruído hospitalar e seus efeitos em funcionários a partir do relato de queixas. Rev CEFAC. 2016;18(6):1379_ 88.

18. Messano GA, Petti S. General dental practitioners and hearing impairment. J Dent. 2012;40(10):821-8.

19. Brasil, Ministério do Trabalho e Emprego. NR 17 - Ergonomia. Portaria MTPS n.o 3.751 , de 23 de novembro de 1990, NR-17 Brasil; 1978 p. 1-14.

20. Fernandes JC, Oliveira JRE de, Fernandes VM. Avaliação do Ruído em Consultórios Dentários. In: XI SIMPEP. Bauru; 2004

21. Silva JLL, Costa FS, Souza RF, Souza JL, Oliveira RS. $O$ ruído causando danos e estresse: possibilidade de atuação para a enfermagem do trabalho. Av EN ENFERMERÍA. 2014;32(1):124-38.

22. Souza TCF, Périssé ARS, Moura M. Noise exposure and hypertension : investigation of a silent relationship. BMC Public Health. 2015;15(328):1-9.
23. Smith SL. The "other " effects of noise. Occup Hazards. 1997;59(1):79-81.

24. Marques FP, Costa EA. Exposição ao ruído ocupacional: alterações no exame de emissões otoacústicas. Rev Bras Otorrinolaringol. 2006;72(3):362-6.

25. Dias A, Cordeiro R, Gonçalves CGO. Exposição ocupacional ao ruído e acidentes do trabalho. Cad Saude Publica. 2006;22(10):2125-30.

26. Pedroso HC, Gonçalves CGO. Percepção e conhecimento dos profissionais da saúde da atenção primária sobre notificação da perda auditiva induzida pelo ruído em Curitiba - Paraná. CoDAS. 2016;28(5):575-82.

27. Paraguay ATT. Perda auditiva induzida por ruído em consultório odontológico [Monografia de especialização]. Recife: CEFAC - Cursos de especialização em fonoaudiologia clínica; 1999, 29p.

28. Cavalcanti TLO, Andrade WTL. Efeitos Auditivos e Extra-Auditivos Decorrentes do Ruído na Saúde do Dentista. Rev Bras Ciências da Saúde. 2012;16(2):161-6.

29. Oliveira ALBM, Campos JADB, Garcia PPNS. Ruído ambiental e sua percepção pelos alunos de odontologia. Rev Odontol da UNESP. 2007;36(1):9-16.

30. Sheldon N, Sokol H. Dental drill noise and hearing conservation. NY State Dent J. 1984 Nov;50(9):557,560-561.

\section{CORRESPONDÊNCIA}

Augusto Henrique Alves de Oliveira

Rua Ricardo Luiz de Andrade, 311 Planalto - Juazeiro do Norte Juazeiro do Norte - Ceará - Brasil

CEP: $63047-310$

E-mail: augustohenrique@leaosampaio.edu.br 